Z. Klin. Chem. Klin. Biochem.

10. Jg. 1972, S. $434-437$

\title{
Quantitative Chrombestimmung im Harn mit flammenloser Atomabsorptions-Spektrometrie
}

\author{
Von K.-H. Schaller, H.-G. Essing, H. Valentin und G. Schäcke \\ Aus dem Institut für Arbeits- und Sozialmedizin und der Poliklinik für Berufskrankbeiten \\ (Direktor: Prof. Dr. H. Valentin) der Universität Erlangen-Nïrnberg
}

(Eingegangen am 22. Oktober 1971/17. Juli 1972)

Sowohl für die Prävention als auch bei bereits bestehenden Gesundheitsstörungen Chrom-exponierter Werktätiger ist der Nachweis des Metalls im biologischen Material von arbeitsmedizinisch-toxikolischem Interesse. Darüber hinaus gewinnen.Chromuntersuchungen auch im Bereich der Stoffwechselforschung zunehmend an Bedeutung. Zur Bestimmung des Chroms im Harn bietet sich als Methode der Wahl die flammenlose A tomabsorptions-Spektrometrie an. Sie ermöglicht im Unterschied zur herkömmlichen Atomisierungsmethode in einer Flamme neben weiteren Vorteilen Metallanalysen ohne vorheriges Aufarbeiten der Proben. Bei 60 beruflich nicht Chrom-exponierten Personen fanden wir eine mittlere normale Chromausscheidung $\mathrm{im}$ Harn von 1,8 1 1,1 $\mu \mathrm{g} / \mathrm{l}$. Als obere Normgrenze errechnete sich eine Chromausscheidung von $4,6 \mu \mathrm{g} / \mathrm{h}, \mathrm{Harn}(\mathrm{p}<0,01)$.

\section{The quantitative determination of urinary cbromium by flameless atomic absorption spectrometry}

For preventive purpose and in view of the disturbances of health already observed in workers exposed to. chromium, the detection of this metal in biological material is of great interest in the field of toxicology in occupational medicine. In addition, general studies on the role of chromium in human metabolism are becoming more important. Flameless atomic absorption spectrometry seems to be the most suitable method for the determination of chromium in body fluids. Unlike customary flame atomizing methods, this method, amongst other advantages, permits the analysis of metals without prior preparation of the specimen. On 60 persons not exposed to chromium we found a mean urinary chromium elimination of $1.8 \pm 1.1 \mu \mathrm{g} / \mathrm{l}$. The upper normal value was $4.6 \mu \mathrm{g} / \mathrm{l}$ urine $(p<0.01)$.

Chrom und seine Verbindungen kommen in vielen industriellen Fertigungsbereichen zur Anwendung. Hier sind insbesondere die Galvanotechnik, die chemische Industrie, die Textilfärberei, die Gerberei und die Zementherstellung zu nennen. Der berufliche Umgang mit diesem Metall kann zu Gesundheitsschäden führen, die pathogenetisch durch die allgemein toxische Schadenspotenz und Kanzerogenität zahlreicher Chromverbindungen sowie deren allergisierende Wirkung bedingt sind (Lit. bei 1). Daher ist sowohl für die Prävention als auch bei bereits bestehenden Gesundheitsstörungen der Nachweis des Metalls im biologischen Material chromexponierter Werktätiger von arbeitsmedizinisch-toxikologischem Interesse.

Die hierfür eingesetzten chemischen Bestimmungsmethoden werden zunehmend durch physikalischchemische Verfahren, insbesondere durch die Atomabsorptions-Spektrometrie ersetzt $(2,3)$. Diese zeichnet sich durch hohe Spezifität, leichte und schnelle Durchführbarkeit und größere Genauigkeit aus $(4,5)$.

In der klassischen Atomabsorptions-Spektrometrie reichen häufig, bedingt durch das Zerstäuber-BrennerSystem, die Nachweisgrenzen zur Metallbestimmung in biologischen Materialien nicht aus. Nach WELz et el. (6) sind für die Durchführbarkeit dieses Analysenverfahrens zwei Voraussetzungen erforderlich.

1. Entweder müssen für geringe Metallkonzentrationen größere Probenvolumina zur Verfügung stehen, so daß aus diesen die benötigten Konzentrationen durch Anreicherungsverfahren gewonnen werden können;

2. oder bei Vorhandensein nur geringer Probenmengen $\mathrm{mu} ß$ das zu analysierende Element in ausreichend hoher Konzentration vorliegen.

Um diese Einschränkungen der Flammen-Atomabsorptions-Spektrometrie zu umgehen, ist es notwendig, anstelle des Zerstäubers andere Systeme zu verwenden. Dabei ging man hinsichtlich einer Dissoziation der Atome neue Wege, indem man die Flamme durch andere Energiequellen ersetzte. Hierfür kommen etwa Hohlkathoden, Graphitöfen oder Laserstrahlen in Betracht (7). Von L'Vov (8) und Massmann (9) sind Kohle- bzw. Graphitrohre als Absorptionsküvetten beschrieben worden. Die Probe wird in flüssiger oder in fester Form in ein Kohle- oder Graphitrohr eingebracht und durch einen elektrischen Lichtbogen bzw. eine elektrische Widerstandsheizung verdampft. Bei schneller Atomisierung kleiner Proben läßt sich erreichen, daß fast alle Atome der Probe in dem nutzbaren Absorptionsvolumen vorhanden sind (9). Auf diese Weise kommt man im allgemeinen mit einem Tausendstel der Konzentration aus, die man bei Verwendung einer Flamme benötigt.

Wie für zahlreiche Spurenelemente und Metalle bietet sich gerade für die quantitative Analyse von Chrom, das nicht nur arbeitsmedizinisch-toxikologisch bedeutsam ist, sondern auch als Metalloenzym eine, essentielle 
Rolle im Intermediärstoffwechsel spielt (10-16), die Methode der flammenlosen Atomisierung an.

\section{Methodik}

Die direkte Analyse des Chromgehaltes im Harn wurde mit dem Perkin-Elmer-Atomabsorptions-Spektrometer 305 vorgenommen, in das anstelle des konventionellen Zerstäuber-Brenner-Systems dic Graphitrohrküvette $\mathrm{HGA}^{1}$ ) 70 eingebaut war.

\section{Graphitrohtküvette}

In einem elektrisch aufheizbaren Graphitrohr, das sich im Strahlengang des Atomabsorptions-Spektrometers befindet, wird die zu analysierende Probe nach kurzfristigem Trocknen und Veraschen durch zeitgesteuerte Temperatureinstellung atomisiert. Im Unterschied zur offenen Flamme des Brenner-Systems verweilen dic freiwerdenden Atome im Graphitrohr oft länger als $1 \mathrm{~s}, \mathrm{~d}$. h. fast tausendmal länger als. in der offenen Flammenströmung. Die Atomisierung im Graphitrohr hat gegenüber dem ZerstäuberBrenner-System mit offener Flamme folgende Vorteile:

1. Vorheriges Aufarbeiten des biologischen Materials entfällt bei vielen Metallanalysen, da Trocknung, Veraschung und Atomisierung durch variable Temperatureinstellung in einem Arbeitsgang erfolgen.

2. Es werden geringere Konzentrationen erfaßt, da im Graphitrohr erheblich mehr Atome zur Lichtabsorption angercgt werden.

3. Zur Analyse ist wesentlich weniger Untersuchungsmaterial erforderlich.

4. Die Analyse leicht oxidbildender Elemente ist wegen der Inertgasatmosphäre im Graphitrohr weniger störanfällig.

5. Durch eine stufenlose Temperatureinstellung kann die optimale Atomisierungstemperatur für jedes Element gewählt werden.

6. Unerwünschte Begleitsubstanzen der Matrix können zeitlich vor der eigentlichen Atomisierung durch stufenweises Erhöhen der Graphitrohrtemperatur zerstört werden.

Abbildung 1 zeigt einen schematischen Querschnitt der Graphitrohrküvette HGA 70. Bezüglich weiterer apparativer Details darf auf die Betriebsanleitung der Herstellerfirma Perkin-Elmer und auf die Veröffentlichung von WeLz et al. (6) verwiesen werden.

\section{Untersuchungsgang}

Als Probenmaterial wurden jeweils $50 \mu$ l eines 24-h-Sammelharns mit einer Eppendorf-Mikropipette durch die Einspritzöffnung der Küvette direkt in das Graphitrohr eingegeben. Entsprechend der
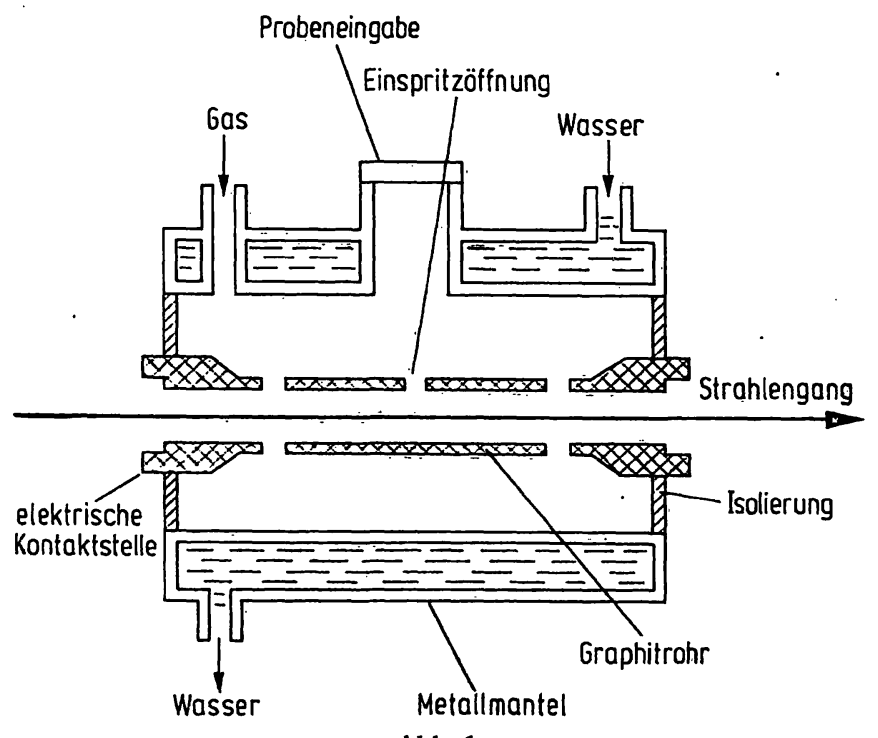

Abb. 1

Schematischer Querschnitt der Graphitrohrküvette HGA 70

1) Heated Graphite Atomizer.
Tab. 1

Angaben für die Temperatur- und Zeitwahl zur Chrombestimmung in Harn

\begin{tabular}{llccc}
\hline \multicolumn{2}{c}{ Temperatur- u. Zeitwahl } & Trocknung & Veraschung & Atomisierung \\
\hline Schritt I & Temperatur & $100^{\circ} \mathrm{C}$ & $1100^{\circ} \mathrm{C}$ & - \\
& Zeit & $120 \mathrm{~s}$ & $30 \mathrm{~s}$ & - \\
\multirow{3}{*}{ Schritt II } & Temperatur & - & $1600^{\circ} \mathrm{C}$ & $2600^{\circ} \mathrm{C}$ \\
& Zeit & - & $30 \mathrm{~s}$ & $20 \mathrm{~s}$ \\
\hline
\end{tabular}

Bedienungsanleitung für die HGA 70 wurde das Arbeitsprogramm 7 gewählt. Die fest eingestellten Graphitrohrtemperaturen bei diesem Programmschritt betragen für den Trockenvorgang $100^{\circ} \mathrm{C}$ und für den Veraschungsvorgang $1100^{\circ} \mathrm{C}$. Getrocknet wurde $120 \mathrm{~s}$ und verascht zunächst $30 \mathrm{~s}$. Da bei dieser Temperaturwahl die unerwünschten Harnkomponenten jedoch nicht vollständig thermisch zersetzt werden, wurde der Veraschungsvorgang mit der zum Atomisieren verfügbaren variablen Graphitrohrtemperatur um weitcre $10 \mathrm{~s}$ erweitert. Hierbei betrug durch Einstellen einer Spannung von 5 Volt die Temperatur etwa $1600^{\circ} \mathrm{C}$. Nach erneuter Veraschung über $30 \mathrm{~s}$ wurde $20 \mathrm{~s}$ bei einer Temperatur von etwa $2600^{\circ} \mathrm{C}$ nach einer Spannungswahl von 10 Volt atomisiert (Tab. 1). Die nach beiden Seiten geöffnete Küvette wurde durch einen konstanten Strom (1,51/min) mit Stickstoff gespült.

Für die atomabsorptions-spektrometrische Endpunktbestimmung wurden folgende apparative Arbeitsbedingungen gewählt:

Wellenlänge: $358 \mathrm{~nm}$

Verstärkung: 5 Umdrchungen

Spaltbreite: $\quad 3$

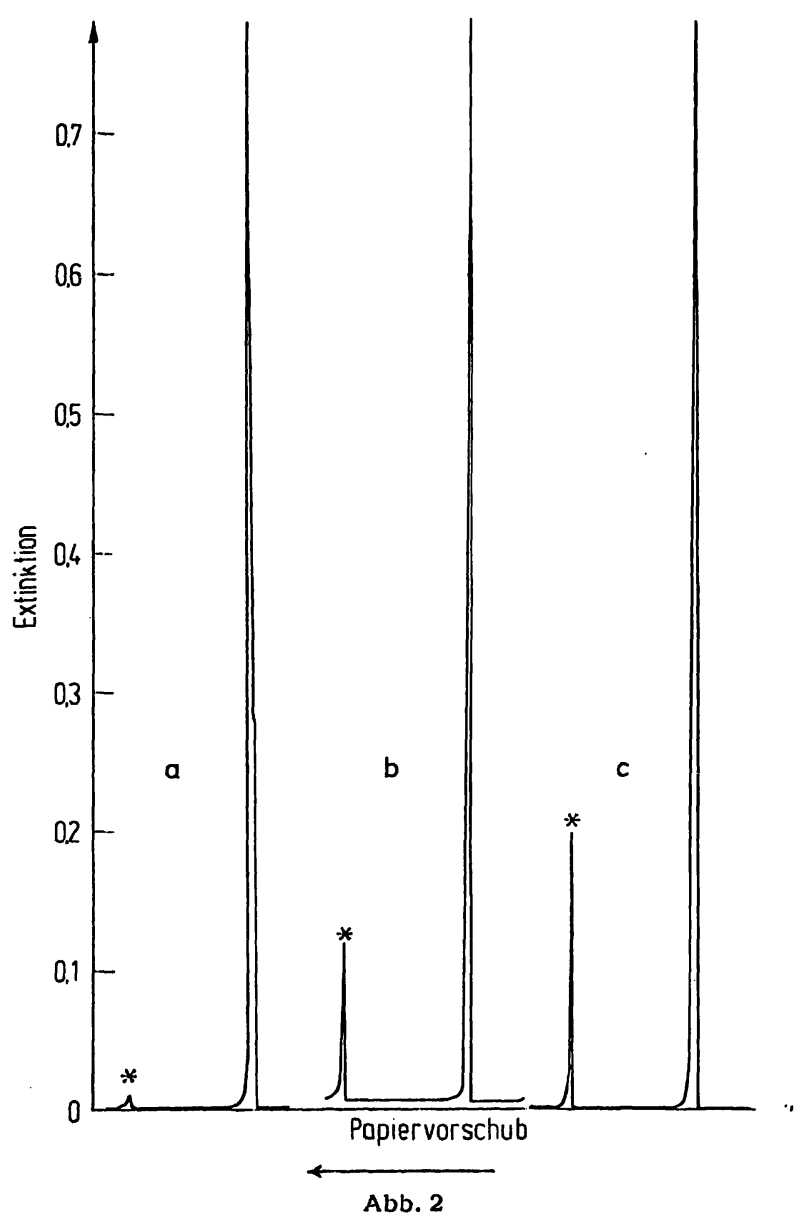

Charakteristische Peaks (*) bei der Chrombestimmung im Harn. Normalurin bzw. Normalurin mit Chromzusatz a) Normalurin, b) Normalurin $+25 \mu \mathrm{g} / \mathrm{l}$, c) Normalurin $+50 \mu \mathrm{g} / 1$ 


\section{Ergebnisse und Diskussion}

\section{Eichkurve}

Als Eichlösungen dienten wäßr. Kaliumchromatlösungen mit bekannten Konzentrationsstufen: $1 \mu \mathrm{g} / 1 ; 5 \mu \mathrm{g} / \mathrm{l}$ und $10 \mu \mathrm{g} / \mathrm{l}$ Chrom. Aufgegeben wurden jeweils $50 \mu \mathrm{l}$. Nach Ausmessen der Peak-Höhe wird an der linearen Eichkurve der Chromgehalt der Harnproben ermittelt. In Abbildung 2 sind die charakteristischen Peaks bei unterschiedlichem Chromgehalt des Harns graphisch dargestellt.

\section{Prüfung der Zuverlässigkeitskriterien}

Die Speziftät einer Elementanalyse mit der Graphitrohrküvette wird u. a. durch Rauch oder weitere nicht spezifische Absorptionen während des Atomisierungsvorganges beeinflußt. Durch das von uns modifizierte Veraschungsverfahren sind jedoch die für eine Rauchentwicklung während der Atomisierung verantwortlichen unerwünschten Harnbestandteile sicher thermisch zersetzt. Dies bestätigten auch vergleichende Untersuchungen mit und ohne Deuterium-Untergrundkompensation ${ }^{2}$ ) (6). Die gemessenen Chrom-Peaks (*) zeigten eine gute Übereinstimmung (Abb. 3).

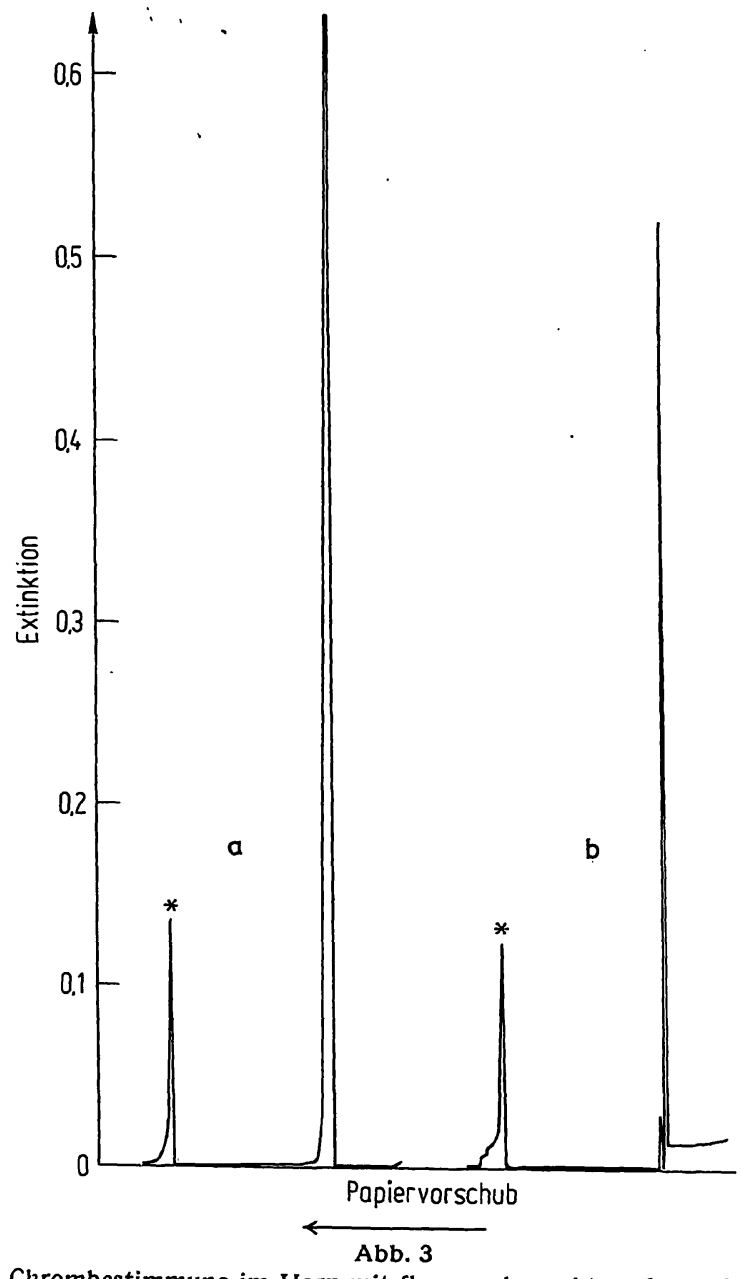

Chrombestimmung im Harn mit flammenloser Atomabsorptions-

a) ohne, b) mit Deuterium-Untergrundkompensation

2) Die Untersuchungen wurden freundlicherweise von, Herrn Dr. B. Welz und Herrn E. Wiedering der Firma Bodenseewerk Perkin-Elmer, Überlingen, durchgeführt.
Zur Prüfung der Ricbtigkeit der Methode wurden Harnproben nicht Chrom-exponierter Probanden mit $5 \mu \mathrm{g} / 1$ Chrom versetzt. Die mittlere Auffindungsrate betrug $93 \%$.

Zur Bestimmung der Präzision in der Serie wurde eine Harnprobe $20 \mathrm{mal}$ analysiert. Bei einer mittleren ChromKonzentration von $6,02 \mu \mathrm{g} / \mathrm{l}$ mit einer Standardabweichung von $0,9^{\circ} \mu \mathrm{g} / 1$ betrug der PeArsonsche Variabilitätskoeffizient $\nu=15 \%$ : Die Präzision von Tag 2u Tag über einen Zeitraum von einer Woche ergab einen Variabilitätskoeffizienten von $\nu=17 \%$.

Die Nachweisgrenze (bezogen auf $1 \%$ Absorption) betrug bei einer Probenmenge von $50 \mu \mathrm{l} 0,2 \mu \mathrm{g} / \mathrm{l}$ Harn bzw. absolut $10 \mathrm{pg}$ Chrom.

\section{Normalwerte}

Von insgesamt 60 beruflich nicht Chrom-exponierten, klinisch gesunden Männern wurden Harnproben auf ihren Chromgehalt analysiert. Bei allen Personen stand der 24-h-Sammelharn zur Verfügung. Die mittlere Chromausscheidung betrug $1,6 \pm 1,1 \mu \mathrm{g} / \mathrm{d}$ und zeigte eine Normverteilung. B̈ezieht man diesen Chromgehalt auf $11 \mathrm{Harn}$, so errechnet sich eine mittlere ChromKonzentration von $1,8 \pm 1,1 \mu \mathrm{g} / \mathrm{l}$. Dieser Wert liegt zwischen dem von ScHROEDER et al. (17) photometrisch mit $0,72 \mu \mathrm{g} / 1$ Harn und dem von Imbus et el. (18) ebenfalls photometrisch mit 3,77 $\mu \mathrm{g} / \mathrm{l}$ Harn angegebenen Ergebnis. Unter Berücksichtigung des von uns bei beruflich nicht Chrom-belasteten Personen ermittelten Normalwertes errechnet sich mit einer Irrtumswahrscheinlichkeit von $\mathrm{p}<0,01$ eine obere Normgrenze der Chromausscheidung von 4,6 $\mu \mathrm{g} / \mathrm{l}$ Harn.

\section{Harnanalysen beruflich Chromexponierter}

Harnproben von 12 in einer galvanotechnischen $\mathrm{Ab}-$ teilung beschäftigten Arbeitern wurden auf ihren Chromgehalt untersucht. Die Ergebnisse der einzelnen Analysen sind in Tabelle 2 dargestellt.

Tab. 2

Ergebnisse von Chromanalysen im Harn zwölf beruflich chromexponierter Arbeiter

\begin{tabular}{|c|c|}
\hline Proband & $\begin{array}{c}\text { Chromgehalt im Harn } \\
{[\mu \mathrm{g} / 1]}\end{array}$ \\
\hline 1 & 15,9 \\
\hline 2 & 7,8 \\
\hline 3 & 6,2 \\
\hline 4 & 24,6 \\
\hline 5 & 7,3 \\
\hline 6 & 8,3 \\
\hline 7 & 3,0 \\
\hline 8 & $5 ; 6$ \\
\hline 9 & 1,4 \\
\hline 10 & 16,0 \\
\hline 11 & 6,1 \\
\hline 12 & 14,0 \\
\hline
\end{tabular}

\section{Praktische Schlußfolgerungen}

Durch den Einsatz der Graphitrohrküvette HGA 70, welche die flammenlose atomabsorptions-spektrometrische Analyse von Spurenelementen und Metallen in 


\section{Neul $\quad \alpha$-Amylase (Diastase)-Bestimmung in Serum und Urin}
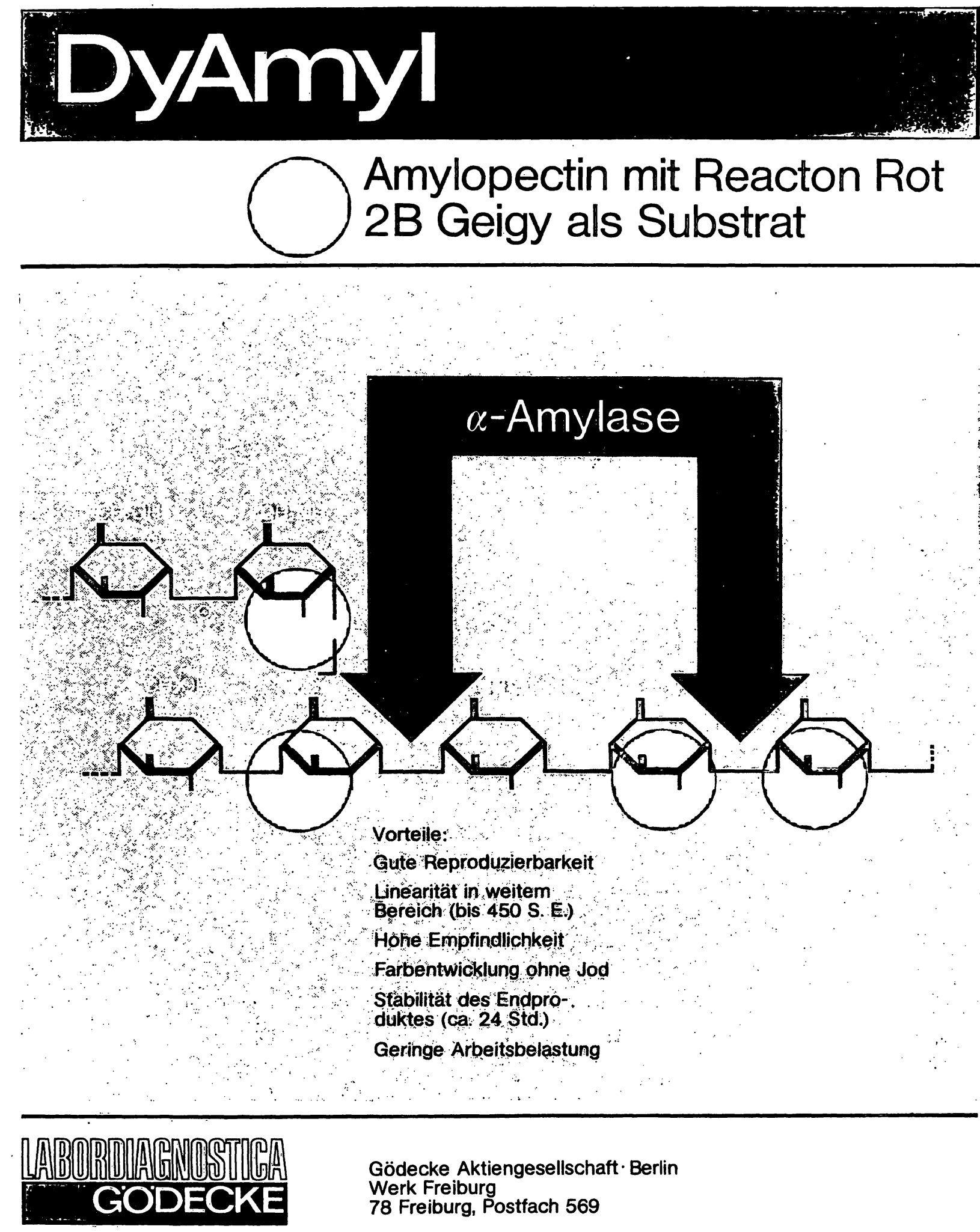

Gödecke Aktiengesellschaft · Berlin Werk Freiburg

78 Freiburg, Postfach 569 


\section{Arzneimittelwirkungen}

Ein Lehtbach der Phatriakologie fith Pharnatrewten, Chemiker und Biologen. Mit eiri führenden Kapitelo in die Anatomie tind Physiologie

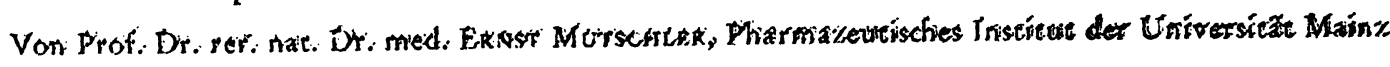

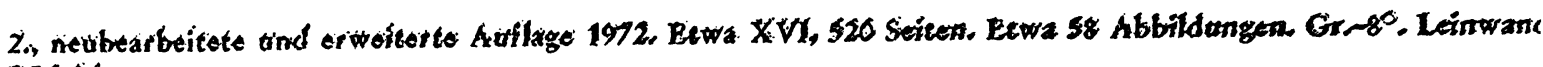
DN 54.-

Vor \%wei Jahren erschieri die 1. Kuflage dieses netren Phatmakologie-Lehrbuches für Pharmanearen, Chemike.

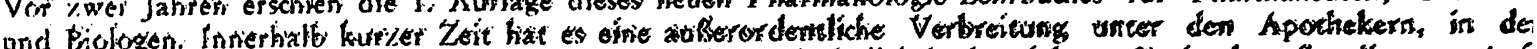

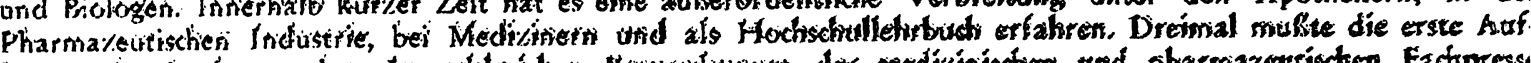

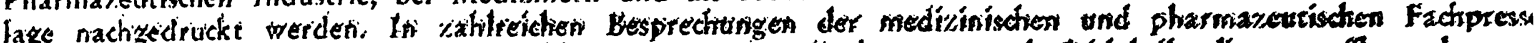

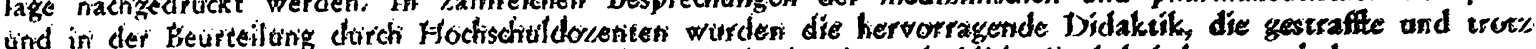

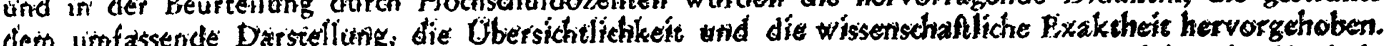

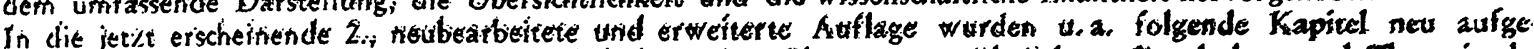

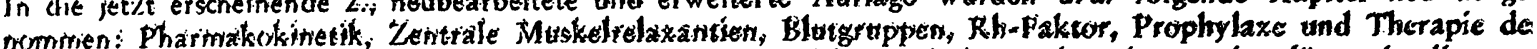
Herlinfariks. Medikamente, die am kespifationstrakt wirksam sind, wurden ebenso eingefüge wie die near Arrineimitelgruppe det Itritrunstiptessiva, Alle ubrigen Kapitel wurden auf den newesten Stand gebrachr, di

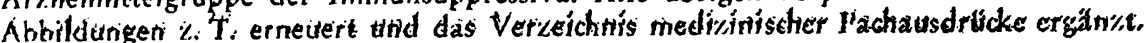

Uiteile der Fichpresse

"Wenti tran vor der Fräge steht; das didaktiseh hervorragende Buch zu empfehlen, so kann man kaum de Wieinung des Auturs beipflichteri, das Buth séi nut litr Pharmateuten, Chemiker und biologen geschrieben. Denr

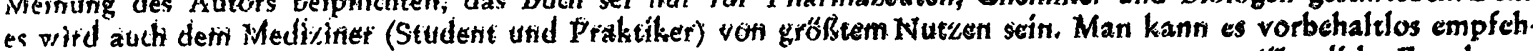
lent:

(Arzeliche Forschung.

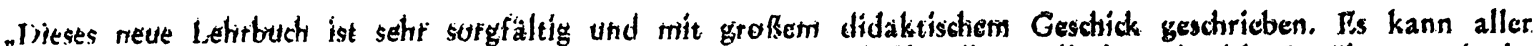
maturwissenschaftlictien Studenten, abef atuch Phatmazeuten und Chemikern, dic irgendwalche Berührung mit de1 Pharmakulogie haberit, voll emipfuhlet werderi."

(Detutsche Medizinische Wodiensdirift;

"The bock should be found tery helpful not only as a thorough introductory text of pharmacology and thera. peutics but also for getheral refereerice use whetever Gettran is read."

(Unlisted Drugs;

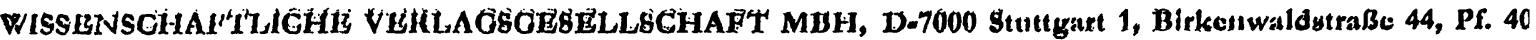

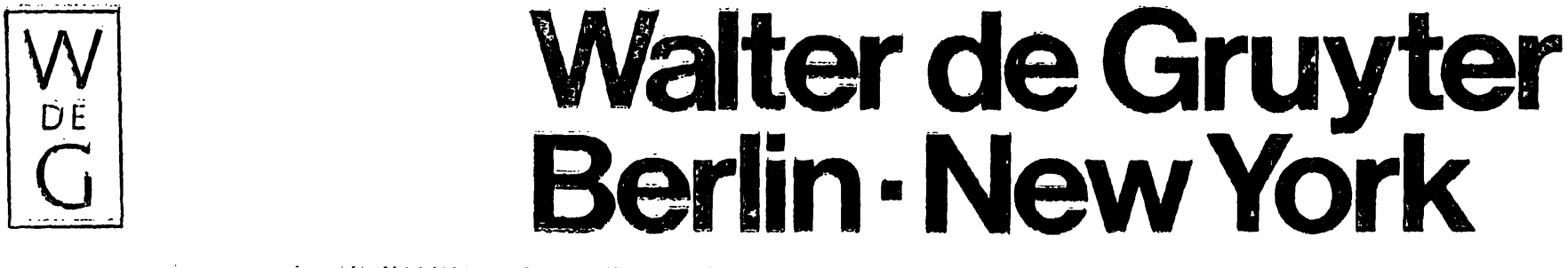

Gitsch - Palmrich

\section{Gynäkologisch-operative Anatomie}

Anliarig: Die Rudiolsolopeh=Rudilkal: opserutloll vör Pról: Er: Hièd: Eduard Gilschi UHd Pliof: Bi: Adolf Hulis Pallinilcli. Mil Geleilwoiteil vón H. Hussleiti Urid I: A firreich. Bilidieil voin Hails Lailg. $21 \times 30 \mathrm{kml}$ villi, 182 Seilleri. Nit 200, $z_{\text {: Tel }}$ melirfurblgell Abbilduligen: 1970 . Gebutte ell blu $160,-=$

ISBIN 311008480 \&

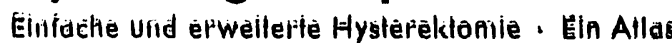

Der Allas sélilietst eline imimer spurbar gewesene Lüukè zwisêhali anutoniszehen Lelirbücher'n und den Darstellungen gyiakóloǵlscher Operationen. Anthand zahlifelcher lllustratlonen Werdefi die dureh die Ojeratianstealınik bedinglen Veränderungen der topos grúplisselieni Analonic des Genillales Und seilier Nachburorgane bei der vaginalen und abdaminalen Hyster ektartle besehrieben. In der Darstellung der Wefilleim'schen Radikaloperation wird besonders der Anleil der Wienter gylläkalagischen Sélivle an dér Weirerentwieklung dieser Methode hervorgehobeh.
In Anhang wird die RadiolsotopenRadikaloperation bolm Kollumkarzinom, die an der I. Universitäts-Fravenklinik Wien entwiekelt wurde, beschrieben und Illustrlert (Typ 1: radioaktives Goldkollold: Typ 2: radioaktlver Phosphor). Sle zelgt dio Möglichkoiten auf, wie man die Resultate der Hysterektomle durch Zusatzmaßnalimen verbessën kann. 
biologischem Material ermöglicht, wird das arbeitsmedizinisch-toxikologische Laboratorium um ein neues, zuverlāssiges Verfahren bereichert. Bei der Bestimmung der Normwerte für Chrom im Harn betrug der Zeitaufwand für eine Einzelanalyse 5 Minuten. Im Unterschied zur Flammen-Atomabsorptions-Spektrometrie erk:lärt sich diese Zeitersparnis dadurch, daß mit der Graphitrohrküvette vor der Atomisierung kein zeitintensives Aufarbeiten des biologischen Materials erforderlich ist. Weiterhin wird damit auch dic Gefahr von Verunreinigung der Proben vermindert. Dieser Gesichtspunkt ist gerade für die Spurenanalyse brw. Lntersuchung von kleinsten Probenmengen bedeutsam.
Während für den Arbeitsmedizincr die Pathogenese der Hypermetallose bei berufsbedingter Chrominkorporation im Vordergrund des wissenschaftlichen Interesses steht, ist für den Stoffwechsclforscher die Frage der Chrommangel-Ausxirkung auf den Organismus aktuell. Auch bezüglich dieser Fragestellung dürfte der Einsat\% der Graphitrohrküvette in Verbindung mit modernen Anreicherungsverfahren wegen der niedrigen Nachweisgrenze der flammenlosen Atomabsorptions-Spcktrometrie im Unterschied zur konventionellen Atomabsorptions-Spektrometrie von methodischem Vorteil sein.

\section{Literatur}

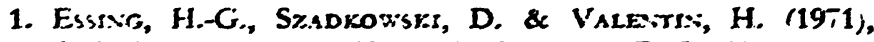
Med. Sachvetst. 67, 35-39. - 2. Feldyas: F. J., Kroeusick, F. C. \& Ptrit, W'. C. (1967), Anal. Chim. Acta 3\%, $489-497 .-$ 3. Yïlki, A. (1971), ZЫ. Arbeitsmed. 4, 122 . - 4. PIRKE, K. M. \& STAMSS, D. (1970), diese Z. 8, 241-248. - 5. LEHNERT, G. \& Schatlffr, K.-H. (1967), Med. Wielt 18, 1131-1133. 6. WeLK, B. \& Wredeknic, E. (19\%0, Z. Anal. Chem. 252, 111-170. - 7. Mexchssser, A. U. (1969), Inaugu:ai-Dissetration, Eriangen. - 8. L'Vor, B. V. (1961;, Spectrochim. teca, Part B, 17, 761. - 9. Mlassssas:, H. (1961), Chimiz 21, 21i-225. - 10. ScrYinPZ, K \& MEPTY, W. (1959), Arch. Birschem. Birphyss. 85, 292-295. - 11. CAsspbes., W. J. \&
MERTL, W' (1963), Amer. J. Phjsiol. 204, 1028-1030. - 12.

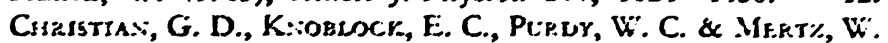
1963;, Birschim. Birsph's. Acta 66, 425-423. - 13. SCHP.DEDER, H. A., BALASIA, J. J. \& V'L:tO:, W. H. j. (1965), J. Nutr. 86, 31-66. - 14. Mert\%, W. 1196T), Fed. Prse. Fed. Amer. Sisc. Exp. Birl. 26, 186-193. - 15. Rorgr:;st, E. E. \& Mrp.T\%, W'. (196-), Fed. Pirse. 26, A batract 255, 301. - 16. Se, hp.jedep, H. A., NAss :- A. P. \& Tisero: 1. H. (19.0), J. Chron. Dis. 23, 123-142. - 17. Sruhroedek, H. A., B.Alasis, J. S. \& Tipto:., S. H. (1962), J. Cr.ron. Dis. 15, 941-264. - 18. Lesels, H. R., CHor.sr, J., MILL.ek, L. H. \& Stersis:G, T. (1963), ircin. Envirs, Health. G, $286-295$.
Prof. Dr. H. Valentin 8520 Eriangea Schillerstr. $25-29$ 